\title{
Homocysteine induces oxidative stress to damage trabecular meshwork cells
}

\author{
ZHI-PENG YOU*, YUE-ZHI ZHANG* , YU-LAN ZHANG, LU SHI and KE SHI
}

Department of Ophthalmology, The Second Affiliated Hospital of Nanchang University, Nanchang, Jiangxi 330006, P.R. China

Received September 19, 2017; Accepted February 8, 2018

DOI: $10.3892 /$ etm.2018.5961

\begin{abstract}
The aim of the present study was to investigate the effect of homocysteine (Hcy) in on human trabecular meshwork cells (HTMCs). A total of 41 patients with primary open-angle glaucoma (POAG) and 53 patients with senile cataracts (control group) were recruited. Plasma and aqueous humor samples were collected and the Hcy concentrations were determined using enzymatic cycling assays. In cell experiments, normal HTMCs were passaged and randomly divided into a blank control group, a normal HTMC group and experimental groups, which were treated with different concentrations of Hcy. The HTMC activities were detected using the Cell Counting Kit-8 method and the HTMC mitochondrial membrane potential (MMP) was detected using JC-1 staining. Reactive oxygen species (ROS) released by trabecular meshwork cells was detected using flow cytometry and superoxide dismjutase-1 (SOD1) expression was detected using immunoblotting. The results revealed that the concentration of Hcy in the plasma and aqueous humor of the POAG group (14.44 \pm 0.86 and $1.60 \pm 0.27 \mu \mathrm{mol} / 1$, respectively) was significantly higher compared with the control group $(10.82 \pm 0.29$ and $0.69 \pm 0.39 \mu \mathrm{mol} / \mathrm{l})$. All tested concentrations $(30,100,300$ and $1,000 \mu \mathrm{mol} / \mathrm{l})$ of Hcy reduced the MMP in HTMCs and inhibited HTMC proliferation in a dose-dependent manner. ROS production by HTMCs significantly increased with increased concentrations of Hcy, whereas SOD1 expression significantly decreased in a dose-dependent manner. In summary, patients with POAG were demonstrated to have
\end{abstract}

Correspondence to: Dr Ke Shi, Department of Ophthalmology, The Second Affiliated Hospital of Nanchang University, 1 Minde Road, Nanchang, Jiangxi 330006, P.R. China

E-mail: 86325294@qq.com

*Contributed equally

Abbreviations: Hcy, homocysteine; HTMCs, human trabecular meshwork cells; POAG, primary open-angle glaucoma; IOP, intraocular pressure; SOD1, superoxide dismutase-1; GSH, glutathione; MMP, mitochondrial membrane potential

Key words: primary open-angle glaucoma, trabecular meshwork cells, homocysteine, oxidative stress, plasma, aqueous humor increased concentrations of Hcy in the plasma and aqueous humor. High concentrations of Hcy in HTMCs induced an oxidative stress state, thereby further inhibiting HTMC proliferation. The results of the present study demonstrate that Hcy may be a potential treatment target in patients with POAG.

\section{Introduction}

Glaucoma is a leading cause of irreversible blindness and it has been predicted that79.6 million people worldwide will suffer with glaucoma by 2020 (1). Primary open-angle glaucoma (POAG) is the most prevalent type of glaucoma worldwide (1). The onset of POAG is associated with a number of factors, including age, sex, race, myopia and diabetes mellitus (2). However, the underlying pathogenic mechanism remains to be elucidated. Elevated intraocular pressure (IOP) is a major risk factor of POAG. The elevation of IOP is caused by a low aqueous humor flow rate resulting from lesions in the aqueous humor outflow pathways (3). The trabecular meshwork, which comprises endothelial-like cells, is the major location of aqueous humor outflow (3). These trabecular meshwork cells are critical for the maintenance of the aqueous humor outflow pathway (4). A previous study reported that patients with POAG have pathological changes in the trabecular meshwork, including a reduced number of human trabecular meshwork cells (HTMCs), an accumulation of extracellular matrix and cytoskeletal changes (5). It has recently been demonstrated that oxidative damage to the trabecular meshwork may serve an important role in the development of POAG (6). It has been reported that the expression of antioxidants, including superoxide dismutase-1 (SOD1) and glutathione (GSH), is increased in trabecular meshwork of patients with POAG (7).

Homocysteine (Hcy) is an intermediate product of methionine metabolism, produced by methionine transmethylation in the human body (8). Hcy causes vascular injury related lesions in vascular endothelial cells during oxidative stress and has been confirmed to be an independent risk factor in the development of cardiovascular diseases (9). The aqueous humor is the flow of blood from the uvea into the ciliary processes; after filtration through reticular capillaries, this fluid is secreted by ciliary epithelial cells (2). As such, the tissue components of aqueous humor are similar to those of plasma, albeit the concentrations are somewhat different. It has been reported that, at high concentrations, Hcy may cause glaucoma due to its oxidative stress function (10). However, the association 
between Hcy and glaucoma remains controversial $(11,12)$. The aim of the present study was to compare Hcy concentrations in the plasma and aqueous humor of patients with senile cataracts and patients with POAG to investigate the effects of Hcy on oxidative stress damage in HTMCs.

\section{Materials and methods}

Clinical information and grouping. All patients were treated at the Second Affiliated Hospital of Nanchang University (Nanchang, China) between January 2014 and December 2015 and provided written informed consent. Patients were divided into the POAG group (patients with POAG) and the control group (patients with senile cataracts). Patients with comorbidities, including hypertension, diabetes mellitus, liver and kidney dysfunction, cerebral infarction or thyroid dysfunction, were excluded from the present study. In the control group, patients with a history of cataracts, eye trauma or eye diseases were also excluded. The POAG group comprised 41 patients (22 men, 19 women; mean age $59.20 \pm 12.8$ years). The control group comprised 53 patients ( 30 men, 23 women; mean age $62.51 \pm 8.49$ years). The present study was performed in accordance with the Declaration of Helsinki and was approved by the Ethics Committee of the Second Affiliated Hospital of Nanchang University.

POAG diagnostic criteria (2). The diagnostic criteria for POAG were as follows: i) IOP $>21 \mathrm{mmHg}$ at any time; ii) fundus examination or optical coherence tomography (OCT) examination revealing glaucomatous optic nerve damage; iii) visual field examination revealing characteristic visual field loss; iv) gonioscope and ultrasound biomicroscope (UBM) examinations revealing an open anterior chamber angle; and v) no signs of secondary glaucoma or a non-glaucomatous cause for the optic neuropathy.

Detection of Hcy in aqueous humor and plasma. A total of $3 \mathrm{ml}$ of fasting blood was collected from study participants and centrifuged $(1,000 \mathrm{xg})$ for $15 \mathrm{~min}$ at room temperature to separate the serum. Serum Hcy levels were measured using an AU-500 automatic biochemical analyzer (Beckman Coulter, Inc., Brea, CA, USA) according to the manufacturer's protocol. The reference range of normal Hcy values was $\leq 10 \mathrm{mmol} / \mathrm{l}$. During trabeculectomy (POAG group) or phacoemulsification cataract surgery (control group), the anterior chamber was punctured to aspirate $0.2-0.3 \mathrm{ml}$ of the aqueous humor and Hcy levels were detected using the AU-500 automatic biochemical analyzer.

Cell culture. Primary HTMCs (cat. no. HUM-iCell-n012) were purchased from iCell Bioscience, Inc. (Shanghai, China). Primary HTMCs were cultured at $37^{\circ} \mathrm{C}$ in Dulbecco's modified Eagle's medium (DMEM; Sigma-Aldrich; Merck KGaA, Darmstadt, Germany) containing $15 \%$ fetal bovine serum (FBS; Gibco; Thermo Fisher Scientific, Inc., Waltham, MA, USA) in an incubator containing $5 \% \mathrm{CO}_{2}$ with $100 \%$ humidity. The culture medium was replaced every 3-4 days. HTMCs were monitored under optical microscope at a magnification of $x 100$ and $x 400$. When cell growth reached $\sim 80 \%$ confluence at the bottom of the culture flasks, cells were dissociated using 1-2 ml 0.25\% EDTA-trypsin, passaged and continuously cultured. Cells at the third and fourth generations were used in the experiments.

Cell proliferation/cytotoxicity assay using Cell Counting Kit- 8 (CCK-8). HTMCs at the logarithmic growth phase were dissociated using $0.25 \%$ EDTA-trypsin to prepare the cell suspension. Cells were diluted using DMEM containing $15 \%$ FBS to a density of $1 \times 10^{4}$ cell $/ \mathrm{ml}$, inoculated into 96 -well plates and cultured in an incubator containing $5 \% \mathrm{CO}_{2}$ with $100 \%$ humidity at $37^{\circ} \mathrm{C}$. When the cell monolayer at the bottom of the wells reached confluence, the culture medium was aspirated and cells were washed 3 times with PBS. The culture medium was replaced and Hcy was added at a concentration of $30,100,300$ or $1,000 \mu \mathrm{mol} / 1$. Vehicle wells contained cells in DMEM supplemented with $15 \%$ FBS, while mock wells only contained DMEM supplemented with $15 \%$ FBS. Each treatment was performed in triplicate. Cells were continuously cultured for $48 \mathrm{~h}$ and washed with PBS, following which $100 \mu \mathrm{l}$ DMEM containing 15\% FBS was added to each well. A total of $10 \mu \mathrm{l} \mathrm{CCK-8} \mathrm{solution} \mathrm{(Sigma-Aldrich;} \mathrm{Merck}$ $\mathrm{KGaA}$ ) was added to each well, samples were thoroughly mixed and the cells were incubated for $4 \mathrm{~h}$ at $37^{\circ} \mathrm{C}$. The absorbance of each well at $450 \mathrm{~nm}$ was measured using a microplate reader.

Detection of intracellular mitochondrial membrane potential (MMP) using the JC-1 method. Following treatment, MMP detection was performed using the JC-1 method (12). Cells were treated with Hcy for $48 \mathrm{~h}$ at $37^{\circ} \mathrm{C}$, washed with PBS, dissociated with trypsin, centrifuged $(1,000 \mathrm{x} \mathrm{g})$ for $15 \mathrm{~min}$ at room temperature and collected. Cells were resuspended in DMEM supplemented with $15 \%$ FBS, mixed thoroughly with $0.5 \mathrm{ml} \mathrm{JC}-1$ staining working solution (Abcam, Cambridge, UK) and incubated at $37^{\circ} \mathrm{C}$ for $20 \mathrm{~min}$. Cells were then centrifuged $(600 \mathrm{x} \mathrm{g})$ at $4^{\circ} \mathrm{C}$ for $4 \mathrm{~min}$, washed twice with ice cold JC-1 staining buffer $(1 \mathrm{X})$ and resuspended in $1 \mathrm{ml} \mathrm{JC}-1$ staining buffer $(1 \mathrm{X})$. The cells were centrifuged $(600 \mathrm{x} \mathrm{g})$ at $4^{\circ} \mathrm{C}$ again for $4 \mathrm{~min}$, resuspended in JC-1 staining buffer (1X) and loaded onto a flow cytometer for qualitative analysis. More than 10,000 cells were observed.

Detection of intracellular reactive oxygen species (ROS). Cells were treated with Hcy for $48 \mathrm{~h}$, dissociated and centrifuged as described above. Cells were mixed thoroughly with $1 \mathrm{ml} \mathrm{DMEM}$ and $1 \mu \mathrm{l}$ DCFH-DA (Abcam) and incubated in a $37^{\circ} \mathrm{C}$ incubator for $20 \mathrm{~min}$. The cells were washed 3 times with serum-free DMEM culture medium and loaded onto a flow cytometer for analysis. More than 10,000 cells were observed.

Detection of SODl expression in cells using western blotting. Cells were treated with Hcy for $48 \mathrm{~h}$, dissociated and centrifuged as described above. The cell pellet containing $5 \times 10^{6}$ cells was mixed with $0.5 \mathrm{ml}$ lysis buffer (Bio-Rad Laboratories, Inc., Hercules, CA, USA), vortexed and suspended. Each $0.5 \mathrm{ml}$ lysis buffer was mixed with $1 \mathrm{ml}$ protein extraction reagent (Bio-Rad Laboratories, Inc.) and centrifuged $(10,000 \mathrm{x} \mathrm{g})$ at $4^{\circ} \mathrm{C}$ for $10 \mathrm{~min}$. The protein layer was extracted, mixed with anhydrous alcohol and centrifuged $(10,000 \mathrm{x} \mathrm{g})$ at $4^{\circ} \mathrm{C}$ for $3 \mathrm{~min}$. The protein pellet 

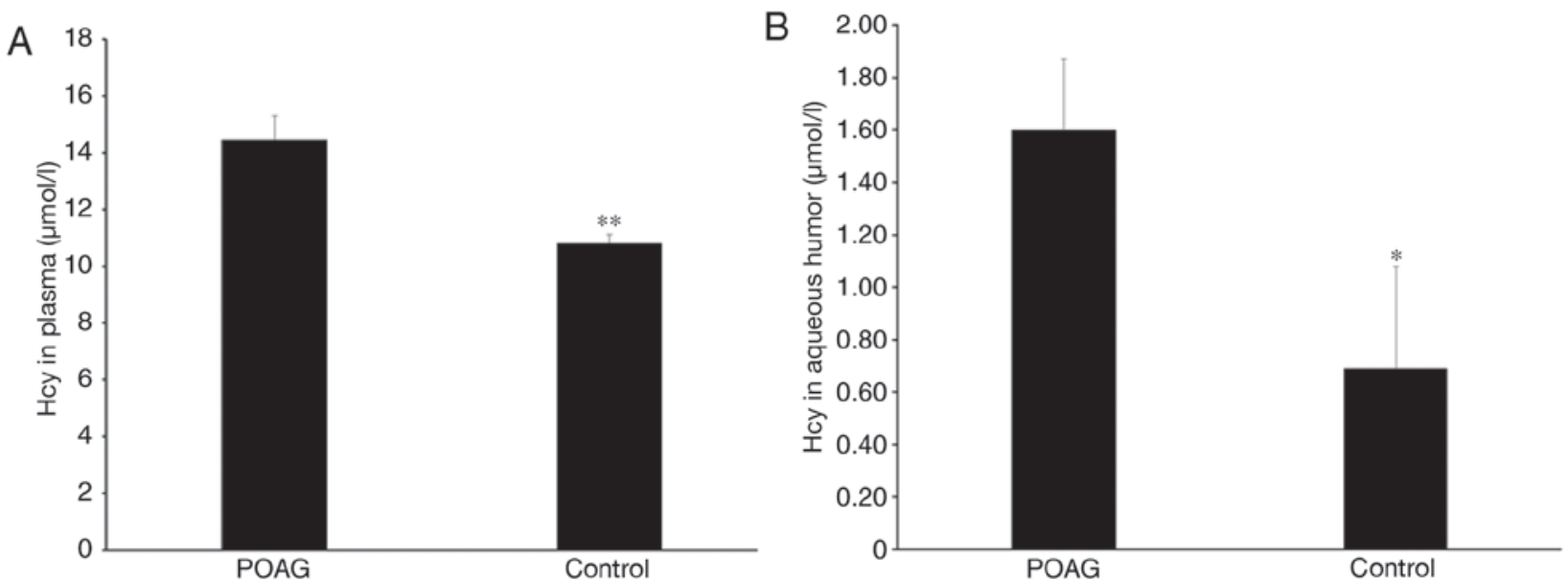

Figure 1. Hcy concentration in the (A) plasma and (B) aqueous humor of patients in the POAG and control groups. " $\mathrm{P}<0.05$ and ${ }^{* *} \mathrm{P}<0.01$ vs. POAG group. Hcy, homocysteine; POAG, primary open-angle glaucoma; Control, patients with senile cataract.

and protein concentration was determined using the BCA method. Equal amounts $(40 \mu \mathrm{g} / \mathrm{lane})$ of protein were separated by SDS-PAGE (10\% stacking gel and 5\% separating gel). Proteins were transferred onto a polyvinylidene difluoride membrane and incubated with the anti-SOD1 primary antibodies (cat. no. sc-271014; Santa Cruz Biotechnology, Inc., Dallas, TX, USA) and anti- $\beta$-actin antibodies (cat. no. ab8226; Abcam) at a dilution of 1:2,000 overnight at $4^{\circ} \mathrm{C}$. $\beta$-actin expression was used to normalize SOD1 expression. The membrane was subsequently washed and incubated with a horseradish peroxidase-conjugated rabbit anti-mouse Immunoglobulin G secondary antibody (cat. no. ab97046; Abcam) with dilution of 1:2,000 for $1 \mathrm{~h}$. Pierce ECL Western Blotting Substrate (Thermo Fisher Scientific, Inc.) was then added in a dark room and subsequent steps performed in accordance with manufacturer's protocol. Gel image analysis was performed using UVP GelDoc-It Imager (UVP LLC, Upland, CA, USA) and the relative densities of the blots were measured by VisionWorks ${ }^{\circledR}$ Image Acquisition and Analysis Software (version 7.0; UVP LLC).

Statistical analysis. Data are presented as the mean \pm standard deviation and were analyzed using SPSS 19.0 (IBM Corp., Armonk, NY, USA). Comparisons between groups were performed using the Mann-Whitney U test. The results of in vitro experiments were compared using the Kruskal-Wallis test followed by a Mann-Whitney post hoc test with Bonferroni correction. $\mathrm{P}<0.05$ was considered to indicate a statistically significant difference.

\section{Results}

Detection of Hcy concentrations in plasma and aqueous humor of patients with POAG. The results demonstrated that plasma Hcy concentrations in the POAG and control groups were $14.44 \pm 0.86$ and $10.82 \pm 0.29 \mu \mathrm{mol} / 1$, respectively. Plasma Hcy was significantly increased in the POAG group compared with patients with senile cataracts $(\mathrm{P}<0.01$; Fig. $1 \mathrm{~A})$. In the aqueous humor, plasma concentrations were $1.60 \pm 0.27$ and $0.69 \pm 0.39 \mu \mathrm{mol} / 1$ in the POAG and control groups, respectively. Compared with the control group, Hcy was significantly upregulated in the aqueous humor of patients with POAG $(\mathrm{P}<0.05$; Fig. 1B).

Growth characteristics of HTMCs and cell proliferation/cytotoxicity assay. Normal HTMCs were generally wide, large and flat, with many processes. A number of morphologies, including star-shaped, spindle-shaped and polygonal-shaped, were observed. Cells overlapped, the cytoplasm was clear and bright and nuclei were large and round or oval-shaped (Fig. 2A and B). During passaging, the cells grew faster during the third and fourth generations and began to become adherent at 10-12 h. Cells were completely attached within $24 \mathrm{~h}$. Generally, cells completely fused to form a closely connected cell monolayer within 1 week. After the 6th generation, the cell growth rate decreased and the cells were spindle-shaped. Furthermore, the cytoplasm-to-nucleus ratio decreased, pigment granule deposition increased, growth speed decreased and the cells were not readily adherent. Therefore, the third to fifth generation of HTMCs were used in the present study.

The cell viability in control wells was considered to be $100 \%$ and cell inhibition rates in the other groups were calculated using the following formula: [optical density (OD) of vehicle wells-OD of experimental wells)/(OD of vehicle wells-OD of mock wells) x 100. The results revealed that cell viability was significantly decreased in all wells treated with Hcy compared with the vehicle ( $\mathrm{P}<0.01$; Table I and Fig. 2C). Hcy appeared to inhibit cell proliferation, with higher Hcy concentrations inducing higher inhibition rates.

Detection of MMP in HTMCs. The JC-1 fluorescent probe, which has an excitation wavelength of $488 \mathrm{~nm}$ and a monomer emission wavelength of $525 \mathrm{~nm}$, is able to enter cells and localize to the mitochondrial membrane (13). Depending on changes in the MMP, the probe emits different colors of fluorescence. In cells with higher MMPs, JC-1 forms an aggregate in the mitochondrial matrix and produces red fluorescence. At lower MMPs, the probe produces green fluorescence. The results of flow cytometry revealed good HTMC activity and normal MMPs in vehicle wells (Fig. 3); in these cells, JC-1 accumulated in the AY2 region and exhibited strong 

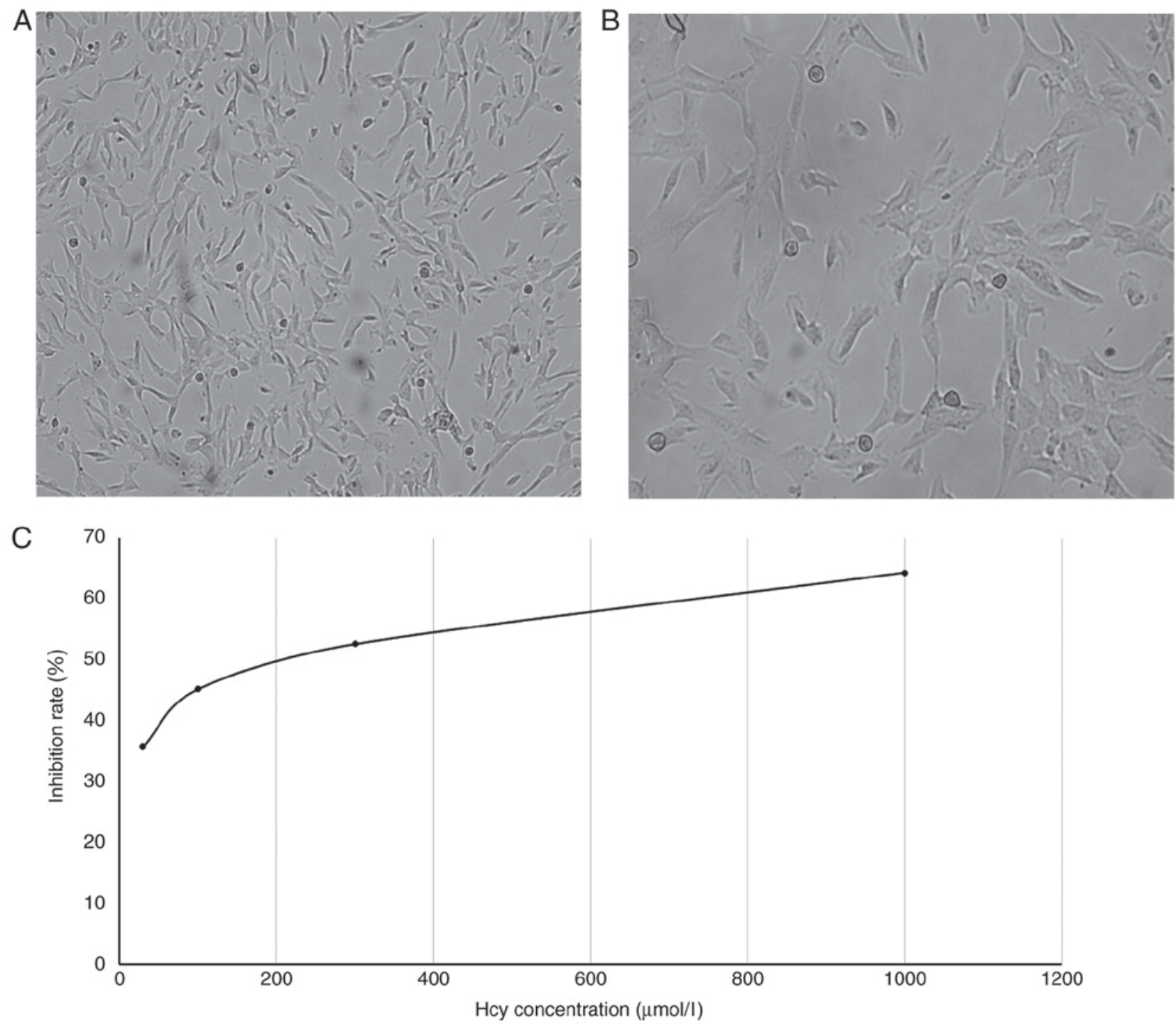

Figure 2. Normal morphology of HTMCs and cell proliferation following Hcy treatment. Morphology of normal HTMCs at passage 3-5, (A) magnification, x100 and (B) magnification, x400. (C) Inhibition of HTMC proliferation following treatment with Hcy. HTMCs, human trabecular meshwork cells; Hcy, homocysteine.

Table I. Effects of Hcy on human trabecular meshwork cells proliferation as assessed using Cell Counting Kit-8.

\begin{tabular}{lcc}
\hline Group & Absorbance & Inhibition rate (\%) \\
\hline Mock & 0 & 0 \\
Vehicle & $1.02 \pm 0.0226$ & 0 \\
Hcy $(\mu \mathrm{mol} / \mathrm{l})$ & & \\
30 & $0.6988 \pm 0.0078^{\mathrm{a}}$ & 35.74 \\
100 & $0.6137 \pm 0.0431^{\mathrm{a}}$ & 45.20 \\
300 & $0.5472 \pm 0.0282^{\mathrm{a}}$ & 52.60 \\
1,000 & $0.442 \pm 0.0516^{\mathrm{a}}$ & 64.31 \\
\hline
\end{tabular}

${ }^{\mathrm{a}} \mathrm{P}<0.01$ vs. Vehicle. Hcy, homocysteine.

red fluorescence. Compared with the vehicle group, JC-1 accumulation in the AY2 area decreased in HTMCs following treatment with Hcy for $48 \mathrm{~h}$; these changes occurred in 71.2,
$55.6,44.8$ and $23.9 \%$ of total cells in the $30,100,300$ and $1,000 \mu \mathrm{mol} / 1 \mathrm{Hcy}$ group, respectively. These results indicated that the concentration of JC-1 aggregates decreased and the MMPs decreased.

Detection of oxidative stress in HTMCs. ROS were detected using flow cytometry and the results demonstrated that the ROS levels in groups treated with $30-1,000 \mu \mathrm{mol} / \mathrm{l}$ Hcy significantly increased compared with the vehicle group $(\mathrm{P}<0.05$; Fig. 4A).

Furthermore, the expression of the oxidative stress marker SOD1 in HTMCs was detected following treatment with different concentrations of Hcy. The results revealed that SOD1 expression in HTMCS decreased following Hcy treatment in a dose-dependent manner (Fig. 4B; P<0.05).

\section{Discussion}

A major risk factor of glaucoma is pathological elevation of IOP (2). Lesions in aqueous humor outflow pathways may obstruct the outflow of the aqueous humor, thereby elevating 

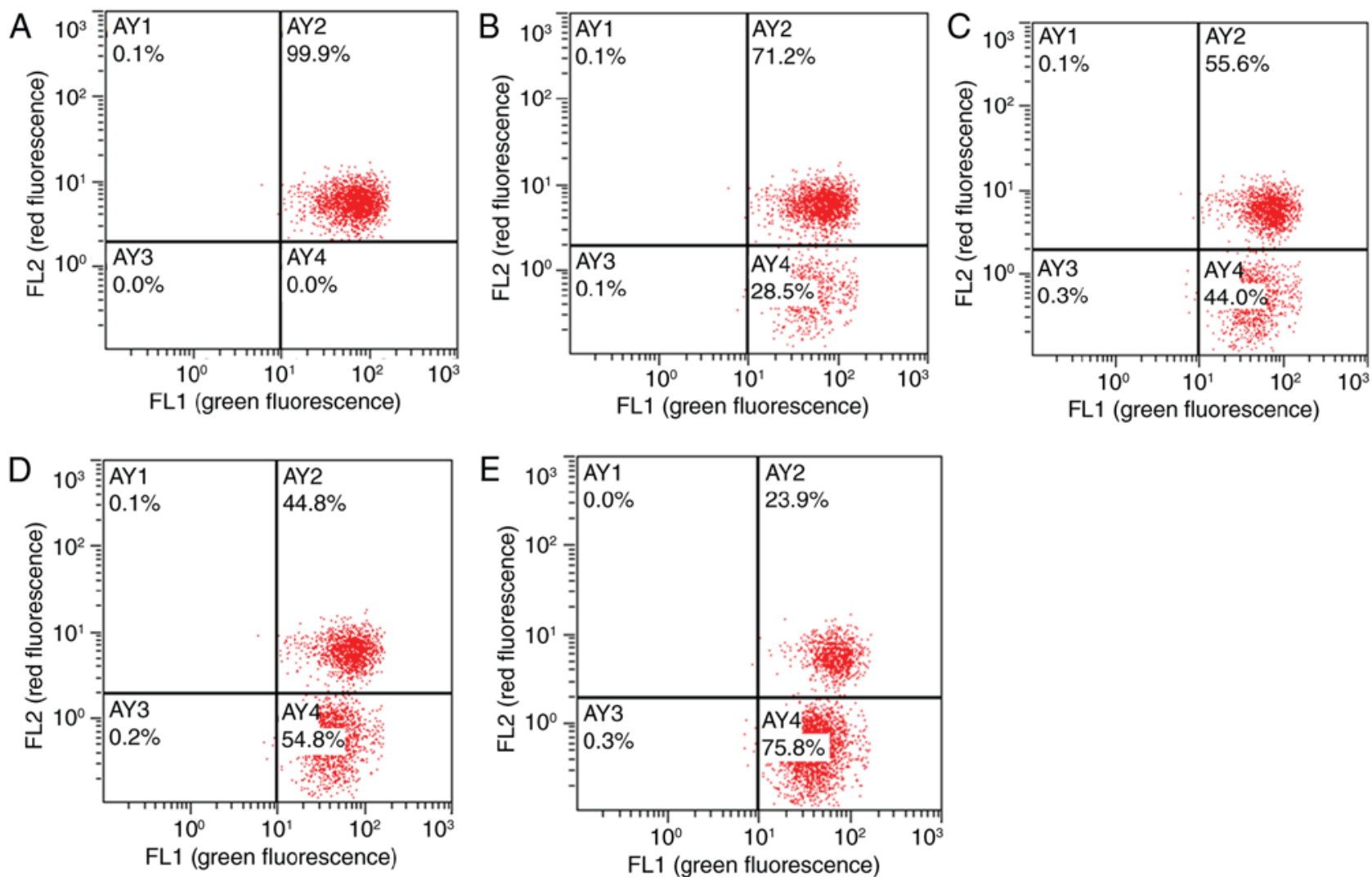

Figure 3. MMP in HTMCs from the (A) blank control group, (B) $30 \mu \mathrm{mol} / 1 \mathrm{Hcy}$ group, (C) $100 \mu \mathrm{mol} / 1 \mathrm{Hcy}$ group, (D) $300 \mu \mathrm{mol} / 1 \mathrm{Hcy}$ group and (E) 1,000 $\mu \mathrm{mol} / 1$ Hcy group. MMP, mitochondrial membrane potential; HTMCs, human trabecular meshwork cells; Hcy, homocysteine.

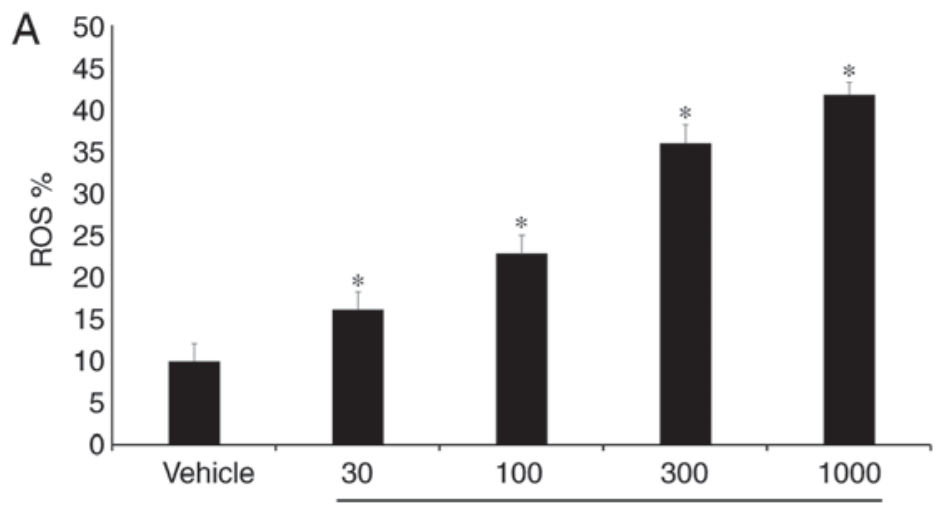

B Hcy $(\mu \mathrm{mol})$
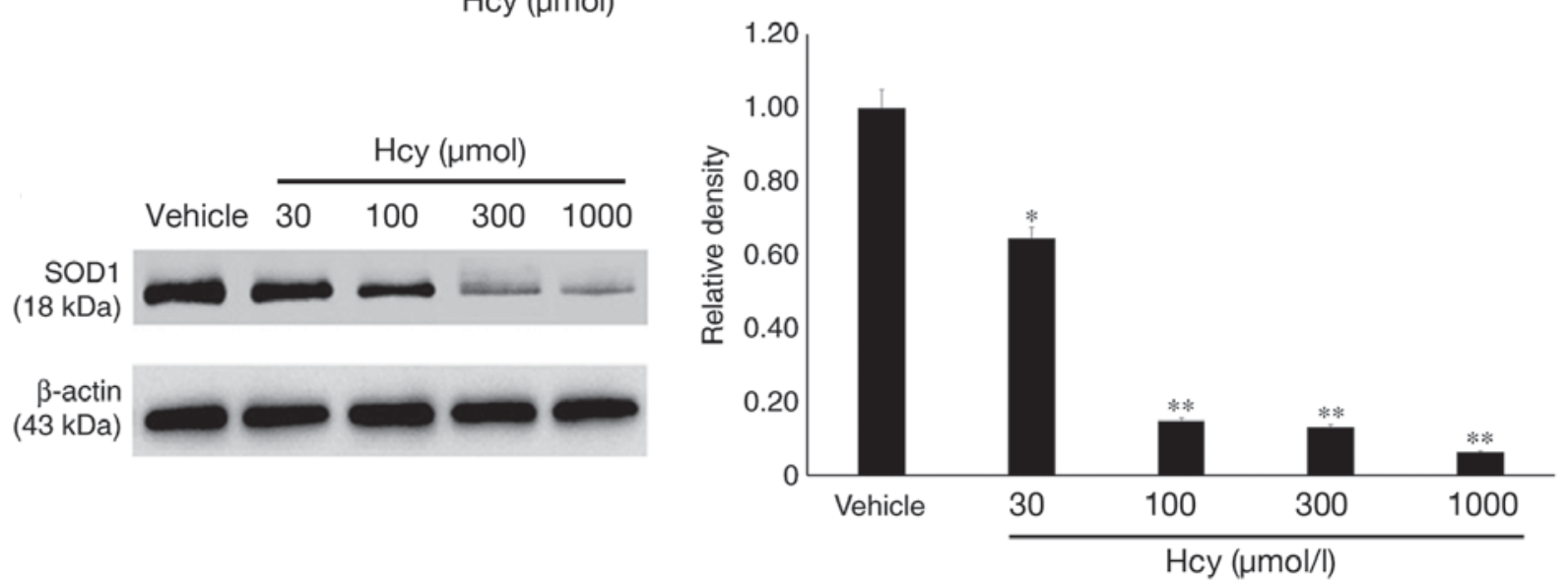

Figure 4. Oxidative stress in HTMCs. Detection of (A) ROS levels and (B) SOD1 expression following treatment with Hcy. ${ }^{*} \mathrm{P}<0.05$ and ${ }^{* *} \mathrm{P}<0.01$ vs. Vehicle. HTMCs, human trabecular meshwork cells; ROS, reactive oxygen species; SOD1, superoxide dismutase-1; Hcy, homocystiene. 
IOP (3). The trabecular meshwork is a major location of aqueous humor outflow (3). Oxidative degeneration of the trabecular meshwork damages the aqueous humor outflow pathways, resulting in insufficient ocular local blood transfusion and, in particular, promoting the degeneration of endothelial cells (6). Early glaucoma is characterized by endogenous cell loss, a reduction of antioxidant levels in the aqueous humor, HTMC apoptosis caused by mitochondrial damage and endothelial cell dysfunction in the anterior chamber (14). A previous in vitro study reported that ROS are able to induce structural changes in the cytoskeleton of trabecular meshwork cells and the extracellular matrix or increase the expression of adhesion molecules, thereby affecting outflow of the aqueous humor and further increasing IOP (15). In addition, it has been reported that all indicators in the aqueous humor, including the total antioxidant capacity, total oxidation value and oxidative stress index, are significantly increased in patients with POAG compared with those with senile cataracts (16).

Hcy is an intermediate product of the methionine cycle pathway and contains a thiol group. When plasma Hcy concentration $>10 \mu \mathrm{mol} / 1$, Hcy may be self-oxidized to produce oxygen free radicals and peroxides, which in turn damage the function and structure of vascular endothelial cells. The results of the present study demonstrated that Hcy concentrations in the plasma and aqueous humor of patients with POAG were significantly higher compared with the control group, suggesting that Hcy is upregulated in patients with POAG. These results were similar to those of Ghanem et al (17). However, other studies reported that there were no statistical differences in Hcy levels of patients with glaucoma and healthy subjects $(18,19)$. The authors hypothesized that this discrepancy may be attributed to ethnic factor since large differences existed in the prevalence and phenotype of POAG among different ethnic groups $(20,21)$. Plasma Hcy levels in patients with senile cataracts in the present study were also slightly higher than $10 \mu \mathrm{mol} / 1$. This result may be associated with a number of factors, including age, a vegetarian diet, Helicobacter pylori infection or increased incidence of atrophic gastritis, all of which may cause malabsorption of coenzymes, including folic acid, VB12 and VB6, which are required for the metabolism of Hcy (22).

In the present study it was also investigated whether treating the trabecular meshwork with high concentrations of Hcy was able to cause structural and functional damage to HTMCs. For healthy adults, Hcy concentrations $>15 \mu \mathrm{mol} / 1$ are considered hyperhomocysteinemia (23). Therefore, based on a study by Perła-Kaján et al (23), an initial Hcy concentration of $30 \mu \mathrm{mol} / 1$ was used in this study. The results demonstrated that cell proliferation was significantly inhibited following Hcy treatment. The inhibition of HTMC proliferation increased in a dose-dependent manner. It has previously been reported that HTMCs treated with $40 \% \mathrm{O}_{2}$ exhibited decreased activity after 10 days (24). This suggests that treating HTMCs with Hcy as an oxidizing agent may induce apoptosis and inhibit cell proliferation.

The JC-1 method was used to evaluate the reduction of MMP, which is used as an early marker of apoptosis (25). The JC-1 fluorescent probe enters cells and accumulates in the mitochondrial matrix to form JC-1 aggregates, which exhibit red fluorescence (26). If the MMP decreases, JC-1 is unable to aggregate in the mitochondrial matrix and remains a monomer that exhibits green fluorescence (26). In the present study, untreated HTMCs exhibited red fluorescent JC-1, whereas cells treated with different concentrations of Hcy exhibited green fluorescent JC-1 that increased in intensity at higher Hcy concentrations. These results indicate that the MMP decreased. It was therefore speculated that Hcy induced the reduction of MMP, thereby damaging cell metabolism and inhibiting HTMC proliferation.

Flow cytometry and immunoblotting were performed to measure ROS levels and the expression of SOD1, which is primarily responsible for $\mathrm{O}_{2}$-clearance (27). As mentioned previously, Hcy treatment increased ROS production in HTMCs. Further investigated revealed that SOD1 expression in HTMCs was downregulated and was significantly negatively correlated with Hcy concentration; these results indicate that Hcy inhibits SOD1 expression. Koh et al (28) used mouse bone marrow cells to demonstrate that the production of ROS increases following Hcy stimulation, while Kang et al (29) reported that increased plasma Hcy concentrations and reduced SOD1 levels were observed in young patients with central retinal vein occlusion. Based on these findings, it was speculated that Hcy may directly induce oxidative stress in HTMCs to cause the excessive production of ROS and promote cell apoptosis and also aggravate the oxidative damage by inhibiting the expression of intracellular antioxidant enzymes.

In summary, the results of the present study demonstrate that plasma and aqueous humor concentrations of Hcy increase in patients with POAG and that elevated Hcy concentrations may be a risk factor of POAG. Excessively elevated Hcy concentrations induce oxidative stress in HTMCs, increase the production of ROS, downregulate the expression of antioxidant enzymes (including SOD1) and reduce MMPs to further inhibit HTMC proliferation. These deleterious effects were also dose-dependent. Hcy may therefore be associated with the onset of POAG. The concentration of SOD1 was used as the oxidative stress marker; as the concentration of SOD1 was not high and the underlying mechanism was not elucidated, there were some limitations in the present study. Future studies should investigate the molecular mechanism, ion channels expression and signaling pathways associated with Hcy-induced oxidative stress in HTMCs.

\section{Acknowledgements}

Not applicable.

\section{Funding}

The present study was supported by the National Natural Science Foundation of China (grant nos. 81460088 and 81760176), the Jiangxi Provincial Training Program for Distinguished Young Scholars (grant no. 20171BCB23092), the Jiangxi Provincial Key R\&D Program (grant no. 20171BBG70099), the Jiangxi Provincial Natural Science Foundation for Youth Scientific Research (grant no. 20171BAB215032) and the Scientific Research Program of Jiangxi Health and Family Planning Commission (grant no. 20171076). 


\section{Availability of data and materials}

The datasets used or analyzed during the current study are available from the corresponding author on reasonable request.

\section{Authors' contributions}

ZPY and KS designed the study and performed the experiments. YZZ and LS performed the experiments. YLZ analyzed the data and KS wrote the manuscript.

\section{Ethics approval and consent to participate}

All patients provided written informed consent. The present study was performed in accordance with the Declaration of Helsinki and was approved by the Ethics Committee of the Second Affiliated Hospital of Nanchang University.

\section{Consent for publication}

The patients provided written informed consent for publication.

\section{Competing interests}

The authors declare that they have no competing interests.

\section{References}

1. Quigley HA and Broman AT: The number of people with glaucoma worldwide in 2010 and 2020. Br J Ophthalmol 90: 262-267, 2006.

2. Kanski J and Bowling B: Clinical Ophthalmology: A systematic approach. 7th edition. Elsevier, London pp3311-3331, 2011.

3. Xin C, Tian N, Li M, Wang H and Wang N: Mechanism of the reconstruction of aqueous outflow drainage. Sci China Life Sci: Dec 22, 2017 (Epub Ahead of Print).

4. Saccà SC, Gandolfi S, Bagnis A, Manni G, Damonte G, Traverso CE and Izzotti A: The outflow pathway: A tissue with morphological and functional unity. J Cell Physiol 231: $1876-1893,2016$

5. Chrysostomou V, Rezania F, Trounce IA and Crowston JG: Oxidative stress and mitochondrial dysfunction in glaucoma. Curr Opin Pharmacol 13: 12-15, 2013.

6. Zhao J, Wang S, Zhong W, Yang B, Sun L and Zheng Y: Oxidative stress in the trabecular meshwork (Review). Int J Mol Med 38 995-1002, 2016.

7. Ghanem AA, Arafa LF and El-Baz A: Oxidative stress markers in patients with primary open-angle glaucoma. Curr Eye Res 35 295-301, 2010.

8. Long Y and Nie J: Homocysteine in Renal Injury. Kidney Dis (Basel) 2: 80-87, 2016.

9. Sanz J, Moreno PR and Fuster V: The year in atherothrombosis J Am Coll Cardiol 62: 1131-1143, 2013.

10. Bleich S, Jünemann A, von Ahsen N, Lausen B, Ritter K, Beck G, Naumann GO and Kornhuber J: Homocysteine and risk of open-angle glaucoma. J Neural Transm (Vienna) 109: 1499-1504, 2002.

11. Xu F, Zhao X, Zeng SM, Li L, Zhong HB and Li M Homocysteine, B vitamins, methylenetetrahydrofolate reductase gene, and risk of primary open-angle glaucoma: A meta-analysis Ophthalmology 119: 2493-2499, 2012.
12. Goyal A, Srivastava A, Sihota R and Kaur J: Evaluation of oxidative stress markers in aqueous humor of primary open angle glaucoma and primary angle closure glaucoma patients. Curr Eye Res 39: 823-829, 2014.

13. Perelman A, Wachtel C, Cohen M, Haupt S, Shapiro H and Tzur A: JC-1: Alternative excitation wavelengths facilitate mitochondrial membrane potential cytometry. Cell Death Dis 3: e430, 2012.

14. Kruk J, Kubasik-Kladna K and Aboul-Enein HY: The role oxidative stress in the pathogenesis of eye diseases: Current status and a dual role of physical activity. Mini Rev Med Chem 16: 241-257, 2015.

15. Babizhayev MA: Biomarkers and special features of oxidative stress in the anterior segment of the eye linked to lens cataract and the trabecular meshwork injury in primary open-angle glaucoma: Challenges of dual combination therapy with $\mathrm{N}$-acetylcarnosine lubricant eye drops and oral formulation of nonhydrolyzed carnosine. Fundam Clin Pharmacol 26: 86-117, 2012.

16. Ergan E, Ozturk F, Beyazyildiz E, Elgin U, Sen E, Cankaya AB and Celik T: Oxidant/antioxidant balance in the aqueous humor of patients with glaucoma. Int J Ophthalmol 9: 249-252, 2016.

17. Ghanem AA, Mady SM, El awady HE and Arafa LF: Homocysteine and hydroxyproline levels in patients with primary open-angle glaucoma. Curr Eye Res 37: 712-718, 2012.

18. Leibovitzh H, Cohen E, Levi A, Kramer M, Shochat T, Goldberg E and Krause I: Relationship between homocysteine and intraocular pressure in men and women: A population-based study. Medicine (Baltimore) 95: e4858, 2016.

19. Zacharaki F, Hadjigeorgiou GM, Koliakos GG, Morrison MA, Tsezou A, Chatzoulis DZ, Almpanidou P, Topouridou K, Karabatsas CH, Pefkianaki M, et al: Plasma homocysteine and genetic variants of homocysteine metabolism enzymes in patients from central Greece with primary open-angle glaucoma and pseudoexfoliation glaucoma. Clin Ophthalmol 8: 1819-1825, 2014.

20. Kim E and Varma R: Glaucoma in latinos/hispanics. Curr Opin Ophthalmol 21: 100-105, 2010.

21. Racette L, Wilson MR, Zangwill LM, Weinreb RN and Sample PA: Primary open-angle glaucoma in blacks: A review. Surv Ophthalmol 48: 295-313, 2003.

22. Wells JL and Dumbrell AC: Nutrition and aging: Assessment and treatment of compromised nutritional status in frail elderly patients. Clin Interv Aging 1: 67-79, 2006.

23. Perła-Kaján J, Twardowski T and Jakubowski H: Mechanisms of homocysteine toxicity in humans. Amino Acids 32: 561-572, 2007.

24. Caballero M, Liton PB, Epstein DL and Gonzalez P: Proteasome inhibition by chronic oxidative stress in human trabecular meshwork cells. Biochem Biophys Res Commun 308: 346-352, 2003.

25. He Y, Ge J and Tombran-Tink J: Mitochondrial defects and dysfunction in calcium regulation in glaucomatous trabecular meshwork cells. Invest Ophthalmol Vis Sci 49: 4912-4922, 2008.

26. Sung DK, Chang YS, Kang S, Song HY, Park WS and Lee BH: Comparative evaluation of hypoxic-ischemic brain injury by flow cytometric analysis of mitochondrial membrane potential with JC-1 in neonatal rats. J Neurosci Methods 193: 232-238, 2010.

27. Izzotti A,Longobardi $M, C$ artiglia $C$ and Saccà SC: Mitochondrial damage in the trabecular meshwork occurs only in primary open-angle glaucoma and in pseudoexfoliative glaucoma. PLoS One 6: e14567, 2011

28. Koh JM, Lee YS, Kim YS, Kim DJ, Kim HH, Park JY, Lee KU and Kim GS: Homocysteine enhances bone resorption by stimulation of osteoclast formation and activity through increased intracellular ROS generation. J Bone Miner Res 21: 1003-1011, 2006.

29. Kang SW, Chang TS, Lee TH, Kim ES, Yu DY and Rhee SG: Cytosolic peroxiredoxin attenuates the activation of Jnk and p38 but potentiates that of Erk in Hela cells stimulated with tumor necrosis factor-alpha. J Biol Chem 279: 2535-2543, 2004.

This work is licensed under a Creative Commons Attribution-NonCommercial-NoDerivatives 4.0 International (CC BY-NC-ND 4.0) License. 